\title{
Reprogramming: faithful reporters
}

\author{
Monya Baker
}

Researchers are finding efficient ways to pick the most promising pluripotent stem cells.

Scientists recognize the cells when they see them: compact colonies with clear edges, and large nuclei that spread almost to the cell membrane. Successful reprogramming experiments make induced pluripotent stem (iPS) cells that resemble embryonic stem cells in both morphology and behavior. Yet appearance can deceive. "I've seen a lot of colonies that look beautiful, but when you test them molecularly and functionally, the colonies are not real, not fully reprogrammed,"

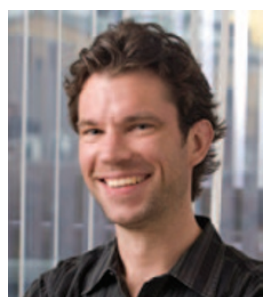

Konrad Hochedlinger at Massachusetts General Hospital says that many beautiful colonies in reprogramming experiments fail to produce high-quality pluripotent stem cells. says Konrad Hochedlinger, a stem cell biologist at Massachusetts General Hospital.

Ultimately, iPS cell lines are assessed with functional tests that require several weeks. For mouse iPS cells, that means generating mouse pups; for human iPS cells, testing for differentiation into all major lineages. These tests are time consuming and expensive, and so researchers need reliable ways to pick the best candidate colonies from a reprogramming experiment, much as an employer will sift through resumes before taking the time to interview job candidates. Surrogate markers of quality are important at several steps: picking which colonies to expand, picking which colony expansions to passage into cell lines, and figuring out which cell lines to submit to the most stringent tests.

\section{Picking colonies}

To make iPS cells, researchers introduce a suite of reprogramming factors into cells, usually by adding genes that encode transcription factors active in pluripotent cells. (A similar approach can be used to make differentiated cells directly; Box 1.) Cells respond to the reprogramming factors by dividing to form colonies, some of which can generate iPS cell lines.

For reprogramming to go to completion, a cell must restart its own pluripotency genes and silence any introduced genes. Often 'partially reprogrammed' cells far outnumber fully reprogrammed ones. These partially reprogrammed cells can closely resemble embryonic stem cell colonies, but they cannot differentiate into the full range of cell types, and their gene expression may be quite different from that of embryonic stem cells.

One way to reduce contamination by partially reprogrammed cells is to halt their growth early. For this, researchers sometimes use reprogramming factors that can be turned on and off at will. The introduced pluripotency genes can be constructed so that they are only expressed in the presence of a small molecule like doxycycline; removing doxycycline from the culture medium will silence them. Fully reprogrammed cells have already activated their own pluripotency genes and so continue to grow, but partially reprogrammed cells do not.

Non-integrating systems, such as Sendai viruses and episomes, offer a similar advantage. Because they are not copied when cells divide, they are diluted out when cells expand, depriving partially reprogrammed cells of pluripotency factors. Growing cells under feeder-free conditions and with fully defined media can also cut down on the growth of partially reprogrammed cells ${ }^{1}$.
Such tools have been commercialized. Several vendors sell reprogramming systems using retroviruses and lentiviruses. A lentivirus produced by Stemgent and sold by Sigma-Aldrich encodes all four of the classic reprogramming factors (Oct4, Sox2, c-Myc and Klf4) in a single construct, with expression activated by doxycycline ${ }^{2}$. Millipore also offers polycistronic lentiviral reprogramming kits. Life Technologies sells a reprogramming kit that includes Sendai virus. And constructs for these and other tools are generally available from the nonprofit plasmid repository Addgene.

Researchers working with mouse cells can use reprogramming systems that allow remarkable control over the transgenes. Separate teams led by Hochedlinger ${ }^{3}$ and by Rudolf Jaenisch ${ }^{4}$ at the Whitehead Institute in Cambridge, Massachusetts have crafted mice in which genes for pluripotency factors are placed under the control of doxycycline and inserted into a genomic locus that is never silenced by methylation and therefore is potentially active in any cell in the mice. These so-called reprogrammable mice allow iPS cells to be generated from any tissue: instead of adding viruses to introduce

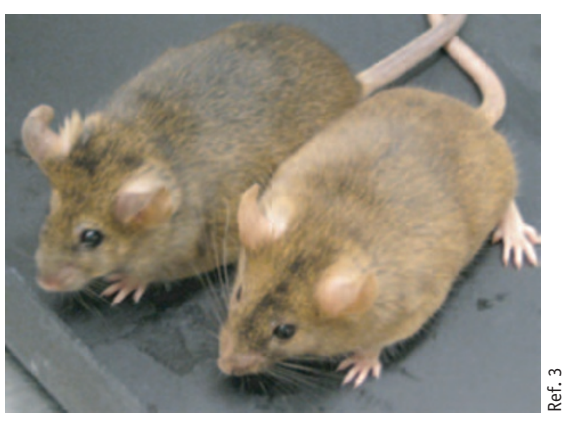

Chimeric mice with all four reprogramming factors in the same genomic locus make producing iPS cells more straightforward. 
reprogramming factors, researchers add doxycycline to activate the ones that are already there.

Using this system creates high yields of high-quality iPS cells, says Hochedlinger: "As soon as you withdraw doxycycline, whatever keeps growing is very much enriched for faithfully reprogrammed cells, so you have much less chance to detect partially reprogrammed cells." Reprogrammable mice are available from The Jackson Laboratory.

\section{BOX 1 FROM SPECIALIZED CELL TO SPECIALIZED CELL}

In 2006, Shinya Yamanaka at Kyoto University showed that a suite of transcription factors could roll back the clock and convert specialized cells to a state much like embryonic stem cells ${ }^{9}$. More recent experiments have shown that the right set of transcription factors can cause a cell to transform directly from one specialized state to another, skipping pluripotency.

The goal is not just showing that cells can change fate: many hope that such conversions could be a faster and potentially safer way of making desired cell types for further study and even cell therapy. Ultimately, researchers will need to find better ways to expand and assess the cells, but for now the field is so new that it hasn't even settled on a name. Various researchers prefer direct reprogramming, next-generation reprogramming, direct conversion or transdifferentiation.

Most studies take the same approach that Yamanaka used to make iPS cells: try lots of transcription factors and painstakingly whittle them down to an essential set. Over the past few years, this has enabled starting populations of fibroblasts to become neurons, cardiomyocytes, blood, muscle and more ${ }^{10}$. Recent work showed that the addition of two cell-specific microRNAs could convert human fibroblasts to neurons capable of action potentials ${ }^{11}$.

Researchers need ways to push more cells completely to the desired fate. In his work to make cardiomyocytes, according to Deepak Srivastava, a cardiologist at the Gladstone Institute in San Francisco, only about 1\% of cells go on to become fullfledged beating cells, but many more go part of the way. "They make sarcomeres, they have calcium transients. They look like cardiomyocytes, but they don't beat," he says, describing a phenomenon observed with getting other cell types to show marks of fully differentiated states.

One strategy may be to screen out factors that maintain cell fate. Last year, Oliver Hobert and colleagues at Columbia University genetically modified Caenorhabditis elegans so that cells would express the transcription factor CHE-1, crucial for the development of a particular type of neuron ${ }^{12}$. Next, they conducted an RNAi screen, knocking down chromatin-associated proteins and scanning the worms for changes. Ultimately, they found a winning combination: without the histone chaperone LIN-53, germ cells in the worm became neurons. "Our approach shows that people should consider loss-of-function approaches," Hobert says.

But Marius Wernig at Stanford University believes that the emphasis is rightly placed on gain-of-function approaches. Factors that modify chromatin state could make cells more flexible, he agrees, "but these can only be supportive, and never be instructive."

And transcription factors are only the most obvious tool, says Sheng Ding, a stem cell scientist at the Gladstone Institute in San Francisco. Small molecules that affect cell signaling pathways may modulate transcription factors, and molecules that modulate metabolism can have surprisingly powerful effects. "Many of the metabolic products are ligands for GPCRs and nuclear receptors for endogenous transcriptional networks and also cofactors for epigenetic enzymes."

In fact, Wernig thinks the biggest barrier may not be in picking the cell of origin or even the right set of transcription factors. Instead, it's knowing how to care for the target cell. "The most impact is how easy it is to grow in cell culture," he says. "There are many cells that just don't like to grow in a Petri dish."
iPS cells can also be made using cells from mice engineered so that endogenous pluripotency genes such as Oct4 or Nanog are tagged with a gene encoding green fluorescent protein. (Often, such mice are crossed with reprogrammable mice or whatever mouse strain a researcher is working with.) If the cells glow green, it shows that they have activated their own pluripotency genes.

Conversely, both human and mouse cells can be reprogrammed with retroviruses encoding pluripotency factors fused with green fluorescent protein. In this case, cells become green in the early stages of reprogramming but shut down fluorescence when the introduced reprogramming factors are silenced. If the reporter fluorescence fades, but the cells continue to grow, they have likely activated their own pluripotency network.

Many researchers want to reprogram cells without modifying their genomes, but non-integrating techniques require more expertise and repeated administration of reprogramming factors.

\section{Staining live cells in situ}

As a general rule, more complex constructs are harder to work with. Thus, many researchers still rely on lentiviruses and retroviruses that produce an abundance of partially reprogrammed cells and so need ways to pick the best colonies. Some scientists have an inherent sense of which cells are faithfully reprogrammed, says George Daley, a stem cell biologist at Children's Hospital Boston, but few are satisfied just to trust their instincts. "You don't want to depend on an intuitive, ineffable set of features," he says. "You want concrete diagnostics."

Three years ago, Daley, Thorsten Schlaeger at Children's Hospital Boston,

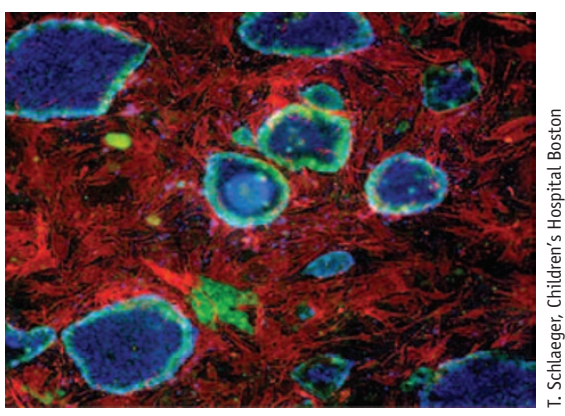

Live-cell imaging of pluripotent stem cells. Red indicates a mouse antibody; blue indicates undifferentiated human pluripotent cells stained with TRA-1-60. 
and others completed a longterm imaging study of reprogramming human cells, the better to identify markers that indicate high-quality pluripotency ${ }^{1}$. They found that several early, oft-used markers, including alkaline phosphatase, SSEA-4, GDF3, hTERT and even Nanog, were unreliable. In contrast, TRA-1-60, a cellsurface marker that appears later in reprogramming, usually held true. "Morphology plus live staining with TRA-1-60 gets you $95 \%$ of the way there," says Daley.

This paper also revealed the utility of in situ live-cell staining, a technique that is slowly gaining traction in the field. Assessing markers in genetically unmodified cells generally means killing or at least disturbing them. Cells are chemically fixed for immunostaining or suspended in dilute solutions for sorting by flow cytometry. In in situ live-cell staining, however, researchers add fluorescent markers directly to growing cells and then wash away unbound tags. This means that the best colonies can be identified without being destroyed.

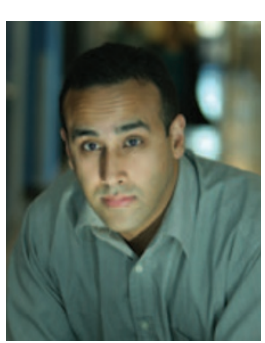

In situ livecell staining also allows researchers to watch colonies over time, which can be important if partially reprogrammed cells have grown over fully repro-

Mick Bhatia at McMaster University warns that iPS cells cannot be trusted until they've been frozen and thawed. grammed ones. "It pinpoints colonies and also gives you an idea of mosaicism," says Mick Bhatia, a stem cell scientist at McMaster University in Ontario.

Several vendors sell antibodies against relevant antigens, but researchers should take care that antibodies are specific and also that they do not perturb the cells, says Bhatia. (TRA-1-60 antibodies seem particularly benign; they bind carbohydrates on a glycoprotein, a trait that may keep them from triggering cell signaling pathways.)

Also, autofluorescence from the culture medium can mask signal from the stains. Schlaeger recommends replacing or
$4 \times$
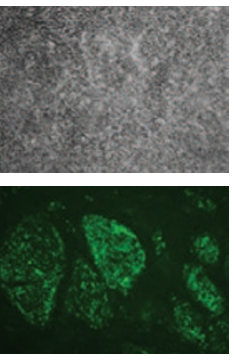

.

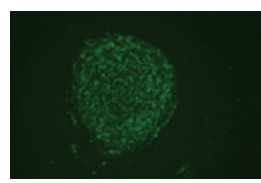

$10 \times$

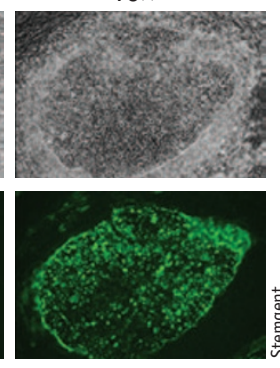

$A-1-60$ diluting the cell culture medium while cells are being imaged. "The cells won't be as happy. But for a short time, it's fine," he says. Using a confocal microscope can also minimize confounding effects, he says.

Some companies are making dedicated antibodies for working with live stem cells; most importantly, these are not stored in sodium azide, a toxic but common preservative. Stemgent, for example, has the 'StainAlive' portfolio. By early April, the company expects to sell antibodies for TRA-1-60, TRA-181, SSEA3 and SSEA4, each available attached to red or green fluorophores. Millipore sells a human iPS selection kit containing antibodies to the pluripotency markers TRA-1-60 and SSEA4, a fibroblast-specific antibody, plus Hoechst 33342 dye, which stains all cell nuclei but is less bright in pluripotent cells.

Live-cell staining is also possible for earlier markers. In January 2012, Life Technologies introduced a small-molecule stain to detect alkaline phosphatase, one of the first markers produced by reprogramming cells and one that some researchers use to pick which colonies to transfer for further observation. Researchers cannot rely solely on alkaline phosphatase staining to identify good iPS lines. Still, such stains, intended to allow cells to continue to grow and develop, can provide a preliminary peek into how well a new reprogramming protocol is working without disturbing the cells, says David Welch, associate director of global market development at Life Technologies.

\section{From colonies to lines}

Picking colonies is just the first step in creating an iPS cell line. But getting many good colonies is a promising start. "If your yield of independent colonies is high, you can breathe easier that the cells are well performing," says Daley. "If you have to squint to find the couple of colonies that might be lurching along, then they may not be pristine."

How to select the best colonies also depends on the experiments the researcher wants to perform next. Bhatia, for example, wants to use iPS cells to make blood. He has shown that colonies producing high levels of c-kit produce blood lineages efficiently; another cell-surface marker, A2B5, indicates that resultant lines will produce neurons efficiently ${ }^{5}$. So now Bhatia uses two stains for his colonies: TRA-1-60 to indicate pluripotency and c-kit to indicate differentiation potential.

After picking colonies, Bhatia starts two more sets of tests: a teratoma assay and differentiation assays. These assessments confirm both pluripotency and differentiation potential. But Bhatia doesn't trust his cells yet. First he must freeze them, thaw them and test them again. "It sounds anal, but you put a lot of work into the clones, and you want to make sure there is no drift," Bhatia explains. No graduate student wants to throw out hard-earned experimental results because some time in the freezer changed cells' behavior. And Bhatia isn't the only one who waits before starting full assessments. Hochedlinger passages his cells eight or nine times, a practice that reduces epigenetic marks that can be carried over from cells' previous identities. In addition, researchers like Bhatia check cells periodically for accrual of cancer-like mutations that can give cells a proliferative advantage.

When reprogramming cells, researchers typically want to end up with at least three 'isolates', or related, well-performing lines that can be studied in parallel and examined for consistency. For experienced

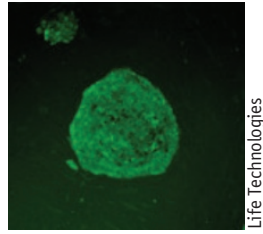

iPS cells treated with a nontoxic smallmolecule stain for alkaline phosphatase researchers using reliable, well-established protocols, four or five colony 'outgrowths' may be sufficient to produce the desired trio. Anyone using a protocol for the first time would be prudent to pick ten or so colonies, says Schlaeger.

Many prefer to start with as many as possible. "We pick as many good-looking 


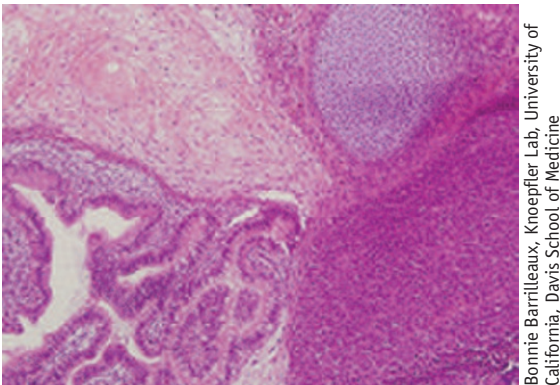

A teratoma from human iPS cells displays many cell types, such as cartilage, connective tissue, glands and brain.

colonies as we can, and see which grow," says James Ellis at the Ontario Human iPS Cell Facility, who is making human disease lines. He reprograms cells using a vector that confers antibiotic resistance as well as a fluorescence marker of pluripotency ${ }^{6}$. That does not mean that every colony outgrowth will produce a good line. Some simply stop growing; others grow too fast, an indication that they are unstable. Even with all the sophisticated markers available, some decisions about which outgrowths are making good lines come down to a judgment call, he says.

\section{Genome-wide tests, slow to take off}

At this point researchers often start doing molecular tests: checking expression of key genes or methylation of important promoters. The goal is to do the cheapest, quickest tests first, so as not to waste resources on cells that don't pan out. Ellis uses qPCR to check for activation of pluripotency genes and silencing of retrovirally added reprogramming vectors, then he does a karyotype to look for large chromosome aberrations and then the lines are subjected to a teratoma assay.

Life Technologies sells a qPCR panel of 96 genes, a set originally established by the International Stem Cell Initiative for evaluating embryonic stem cells. Millipore and others have pluripotency panels that evaluate expression of a dozen or so genes. Academic researchers have also put forward evaluation tools based on genome- wide expression. These include a so-called scorecard to estimate differentiation potential $^{7}$ and the online tool Pluritest ${ }^{8}$, which compares gene expression data from a newly derived cell line to that of other pluripotent cell lines.

But although researchers are generally aware of comprehensive tests, few are using them, especially as they are already performing functional assays. Measuring the expression of hundreds of genes is a lot of work, says Hochedlinger. "Unless we can reduce the genes to five or ten, I doubt this will be a widely used approach to systematically test iPS colonies."

The practice of stem cell genomics may soon be expanding, however. The California Institute for Regenerative Medicine recently pledged $\$ 40$ million to launch one or two dedicated centers. And Life Technologies is convinced that its Personal Genome Sequencer will encourage more researchers to perform genomewide expression and methylation studies.

Nonetheless, the gold-standard assays remain functional tests. Currently the teratoma assay is considered (with some debate) to be the most stringent test for human cells. This involves injecting cells into a mouse and waiting to see whether they generate a tumor containing cells representing the major tissues. Most researchers perform this test, all the while hoping that molecular tests will eventually replace it.

"No one is really comfortable not doing the teratoma assay," says Paul Knoepfler, a stem cell biologist at University of California, Davis Medical School. It is one thing to see expression levels of genes, but something else entirely to see a collection of specialized cells in a tumor: brain, bone, cartilage and more. The teratoma test is not a perfect assay, he says, but he thinks people will continue doing it for years to come. "It's so reassuring to see the pluripotency come to life."

Not everyone agrees. If a cell line forms embryoid bodies, passes differentiation tests and expresses expected markers, it is pretty much guaranteed to make a teratoma, says Schlaeger, whose center has generated hundreds of iPS cell lines. Important, surprising findings and new protocols may require "every assay that can be thrown at the cells," he says, but routine studies may not require such a high bar. "If a lab is proficient at generating iPS cells and has just produced 20 new Parkinson's lines that passed all in vitro tests of pluripotency and showed virtual equivalence to ES cells, it should be sufficient to only perform the teratoma test on a few representative lines, if at all."

"It's a faulty and antiquated assay," agrees Daley. It was the best available during the development of the field, he says, but the assay is not quantitative, and few labs perform it rigorously, by ensuring that teratomas actually produce all the important lineages. At least for lines produced via well-established protocols, the field will move to molecular tests eventually, he predicts. When that will happen, and what exactly those tests will be, is still unclear. But the early assessments that identify well-performing pluripotent lines are clearly key. Researchers may spend years of work with follow-up experiments on these cell lines, so the starting materials require rigorous assessment.

\section{Monya Baker is technology editor for Nature and Nature Methods \\ (m.baker@us.nature.com).}

1. Chan, E.M. et al. Nat Biotechnol. 27, 1033-1037 (2009).

2. Carey, B.W. et al. Proc. Natl. Acad. Sci. USA 106, 157-162 (2009).

3. Stadtfeld, M., Maherali, N., Borkent, M. \& Hochedlinger, K. Nat. Methods 7, 53-55 (2010).

4. Carey, B.W., Markoulaki, S., Beard, C., Hanna, J. \& Jaenisch, R. Nat. Methods 7, 56-59 (2010).

5. Hong, S.H. et al. Cell Stem Cell. 9, 24-36 (2011).

6. Hotta, A. et al. Nat. Methods 6, 370-376 (2009).

7. Bock, C. et al. Cell 144, 439-452 (2011).

8. Müller, F.J. et al. Nat. Methods 8, 315-317 (2011).

9. Takahashi, K. \& Yamanaka, S. Cell 126, 663-676

10. Vierbuchen, T. \& Wernig, M. Nat. Biotechnol. 29, 892-907 (2011).

11. Yoo, A.S. et al. Nature 476, 228-231 (2011).

12. Tursun, B., Patel, T., Kratsios, P. \& Hobert, 0. Science 331, 304-308 (2011). 